\title{
DIE SPRICHWÖRTLICHE REDENSARTEN
}

\author{
Huseynzade Khoshgadam \\ Azerbaijan State Oil and Industry University \\ DOI: https://doi.org/10.31435/rsglobal_ws/12072018/6016
}

\section{ARTICLE INFO}

Received: 10 May 2018

Accepted: 22 June 2018

Published: 12 July 2018

\section{KEYWORDS}

\section{proverbs;}

vocabulary;

outstanding;

sayings;

experience;

verdict;

embrace;

traits.

\begin{abstract}
In this article we are talking about the phraseology of proverbs in German and Azerbaijani. What are proverbs? - These are a set of complete phrases created by the people, tested by experiments and time, briefly and clearly expressed in the public. "Love does not get old"(Alte Liebe rostet nicht.), "The Word of Men is the Law"- «Ein Mann, ein Wort». That concrete and abstract proverbs retain the meaning and essence of the sentence as distinct from riddles and idiomatic expressions.

They correspond to the phraseological compounds of V. Vinogradov's classification, are an integral part of the language and enrich the vocabulary of the German language. This classification is based on a functional principle and therefore they can be applied both in style and in lexicology. This article deals with word combinations, phraseological units and compounds, idiomatic expressions, winged phrases and proverbs.
\end{abstract}

Citation: Huseynzade Khoshgadam. (2018) Die Sprichwörtliche Redensarten. World Science. 7(35), Vol.3. doi: 10.31435/rsglobal_ws/12072018/6016

Copyright: (C) 2018 Huseynzade Khoshgadam This is an open-access article distributed under the terms of the Creative Commons Attribution License (CC BY). The use, distribution or reproduction in other forums is permitted, provided the original author(s) or licensor are credited and that the original publication in this journal is cited, in accordance with accepted academic practice. No use, distribution or reproduction is permitted which does not comply with these terms.

Der Bilderreichtum einer Sprache offenbart sich auch in den sprichwörtlichen Redensarten. Wir kennen die Bildhaftigkeit des Ausdrucks bereits als ein hervorstechendes Merkmal der volkstümlichen rede, und tatsächlich ist der Ursprung der meisten bildhaften Redensarten im Volksmund zu suchen. Sehr viele von ihnen kommen aus den Sonderwortschätzen und der speziellen Phraseologie der verschiedenen sozialen Gruppen unseres Volkes den sog. Standes-, Berufs-, und Fachsprachen. Viele bildhafte Redensarten sind heute noch plastisch und anschaulich, so dass ihre Bilder für jeden Sprachgenossen verständlich sind, andere dagegen sind nicht mehr ohne weiteres zu durchschauen.

Die Erklärung mancher Redensarten stößt auch deshalb auch Schwierigkeiten, weil sie im Volksmund vielfach Umdeutungen, Vermischungen und Verstümmelungen unterworfen waren, die das Verständnis erschweren.

Die Redensart $j-m$ einen Bären aufbinden ist durch Vermischung der Wendungen $j-m$ etwas aufbinden und einen Bären anbinden (Schulden machen) entstanden. Andere bildhafte Redewendungen aus dem Bauernleben, die sprichwörtlich geworden sind, sind das Pferd am Schwanze aufzäumen (etwas verkehrt machen), den Stier bei den Hörnern packen, wie der Ochs vor dem neuen Tor stehen, gut beschlagen sein, im Gänsemarsch gehen, die Ohren spitzen, wie Hund und Katze miteinander leben, auf den Hund kommen (-herunterkommen in schlechte Verhältnisse geraten).

Was ist ein Sprichwort? Ein Sprichwort ist ein im Volke entstandener und im Volksmunde lebender, in knapper Form ausgesprochener Erfahrungssatz („Alte Liebe rostet nicht") oder es ist ein Urteil, eine Meinung, die belehren, mahnen oder warnen will. („Ein Sperling in der Hand ist besser als eine Taube auf dem Dach").

Dieser als Erfahrung oder Urteil des ausgesprochenen allgemeinen Satzes, dieses Axiom der Volksphilosophie hat sehr oft die Form eines Bildes („Hunger ist der beste Koch“). Solche Allegorien sind bei allen europäischen Völkern beliebter als die abstrakten, bildlosen Weisheitssätze. Jedes Sprichwort, mag es abstrakt oder im Gewande eines Gleichnisses auftreten, enthält in einem Satz 
einen selbständigen Gedanken und unterscheidet sich dadurch von der Redensart und der Redewendung. Dies sind in der Regel keine selbständigen Sätze, denn sie enthalten statt einer wirklichen Aussage lediglich einen bildlichen Ausdruck, sei der nun ein Vergleich, eine Metapher, Hyperbel oder ein Paradoxen. Es sind Redefiguren, also stilistische Begriffe, wie z. B. die Redensarten: „Hunger haben wie ein Wolf“.

Es ist für das Sprichwort kennzeichnend, dass es einen allgemeinen Gedanken, eine allgemeine Beobachtung oder Erfahrung an einem konkreten Einzelfall anschaulich, sinnbildlich darstellt. So wird die altbekannte, immer wieder bestätigte Beobachtung, dass die Kinder in die Art ihrer Eltern schlagen, im Sprichwort so abstrakt und sachlich formuliert, sondern durch ein Bild veranschaulicht: Der Apfel fällt nicht weit vom Stamme. Ot kökü üstə bitər.

Die bildhafte Ausdruck dient dem Sprichwort also zur volkstümlichen Versinnlichung eines abstrakten Gedankens. Betrachtet man die Sprichwörter mit bildhaftem Charakter genauer, so kann man zunächst zwei große Gruppen unterscheiden. Die erste umfasst die Sprichwörter, die als Ganzes ein Bild darstellen, die nach den Worten Seilers „vollkommen und ohne Rest in konkrete Anschauung verwandelt" sind, z. B. die folgenden Sprichwörter, die eine treffende

Darstellung recht unerfreulicher Erscheinungen der Klassengesellschaft geben: Dem Esel, der das Korn zur Mühle trägt, wird die Spreu. - Eşşək işləyir, at yeyir. Der Fisch fängt am Kopf an zu stinken- Balıq başdan iylənər. Wenn man die Treppe wäscht, muss man von oben anfangen -İş görəndə dibdən görmək lazımdır. Wenn man den Hund schlagen will, so hat er Leder gefressen- İti öldürənə sürütdürərlər. Wenn dem Esel zu wohl ist, geht er aufs Eis tanzen und bricht ein Bein. -Dəvə oynayanda qar yağar.

Es ist dafür gesorgt, dass die Bäume nicht in den Himmel wachsen; stille Wasser sind tief; Neue Besen kehren gut; In kalten Öfen bäckt man kein Brot: Mit Speck fängt man Mäuse; Man muss das Eisen schmieden, solange es heiß ist; Allzu scharf macht schartig.

Bei der zweiten Gruppe erstreckt sich das Bild nur über einen Teil des Sprichwortes, während der Rest unbildlich bleibt. In den meisten Fällen erscheint das Bild im Prädikat, während das Subjekt direkt und unbildlich bezeichnet wird.

Diese Sprichwörter geben in der Regel einen Vergleich zwischen einem abstrakten Begriff und einem konkreten Ding: Hoffnung ist ein Schiff mit einem Mast von Stroh; Faulheit ist der Schlüssel zur Armut; Freundschaft ist des Lebens Satz; ein gutes Gewissen ist ein sanftes Ruhekissen.

Auch im Sprichwort tritt die Tierklasse auf:

Der Neid ist eine Natter; Die Hoffart steckt den Schwanz übers Nest; Die Armut ist des Reichen Kuh; Der Geiz ist ein Ross, das Wein fährt, und Wasser säuft; Eine gezähmte Zunge ist ein seltener Vogel.

Von den Sprichwörtern zu scheiden sind auch die moralisierenden Sinnsprüche, Sinngedichten, Sentenzen und Aphorismen, die nach weislich aus der Feder eines Schriftstellers stammen. Freilich ist auch das Sprichwort einmal im Munde eines Einzelnen entstanden: die unbekannten Einzelnen aber pflegt man als Volk zu bezeichnen und ihre Sprüche sind volkstümlich. Wenn die Aussprüche bekannter dichter nicht zu Sprichwörtern geworden sind, so liegt das vielleicht an der Denk- und Ausdrucksweise des Autors, die der Volkstümlichkeit entbehrt.

Manches Sprichwort, unter die historische Lupe genommen, wird sich als geflügeltes Wort einer älteren Zeit erweisen. Dem internationalen Sprichwort „Eine Schwalbe macht noch keinen Sommer"- "bir gül ile bahar olmaz", sieht man seinen altgriechischen, literarischen Ursprung nicht an, und für das Wort „Aus den Augen, aus den Sinn“ - „Gözdən uzaq, könüldən iraq“ vermittelt der Deutsche schwerlich eine schriftliche lateinische Quelle.

Aus der Gegenüberstellung der Sprichwörter der beiden Sprachen ist ferner ersichtlich, dass jedes Volk in seinem Sprichwörterschatz vielfach eine eigene Formgebung hat für Erfahrungssätze und ethische Begriffe, dass aber die Bewertung der Tugenden und Laster im großen und ganzen übereinstimmend ist. Man muss sich davon hüten, aus einer Anzahl von Sprichwörtern ablesen zu wollen, das ein Volk höhere moralische Qualität als das andere besitzt, denn aller Völker, darunter auch das deutsche und das aserbaidshanische Volk, unterscheiden scharf zwischen Recht und Unrecht, Wahrheit und Lüge.

Eine allgemein geschätzte gute Eigenschaft mag im Spruchschatz des einen Volkes eine liebevollere Behandlung gefunden haben als bei dem Nachbarvolk. Ebenso kann eine Untugend sprichwörtlich vom im Westen schärfer gegeißelt werden als von dem Volk im Osten, oder umgekehrt, der Unterschied ist stets nur ein gradueller, die Einstellung im Grunde die Gleiche.

Es lässt sich aus einem Sprichwort nicht ohne weiteres auf den Volkscharakter schließen, wie das heute schulmäßig immer noch geschieht. Denn eine beträchtliche Menge von Sprichwörtern ist international, zum mindesten gesamteuropäisch. Die Sprichwörter wandern bekanntlich gleich 
Märchenmotiven von Volk zu Volk, und darum hat jede Sprache mehr oder weniger Entlehnungen Lernspruche - aufzuweisen.

Beide Völker dürfen stolz sein auf ihren Spruchreichtum. Es muss aber eingeräumt werden, dass sich des Deutschen Verhältnis zu seinem Sprichwort im Laufe der Jahrhunderte gewandelt hat. An der Schwelle der Neuzeit, aber auch noch im 16. Und 17. Jahrhundert; war das gute alte deutsche Sprichwort in aller Munde. Heute findet die «Weisheit auf der Gasse" viel seltener Anwendung als ehedem.

Über die Entstehungszeit, mindestens aber über die Zeit der Verbreitung der deutschen Sprichwörter sind recht gut unterrichtet. Ein beträchtlicher Teil des deutschen Sprichwörterschatzes hat schon im Mittelalter seine feste Prägung erhalten und einzelne Sprüche lassen sich bis ins 10.Jh.hinauf verfolgen. Den Untersuchungen Fr. Seilers (1922) und besonders S. Singers (1944 - 47) verdanken wir weitgehenden Aufschluss über die Anfänge des deutschen Sprichwortes in seinem lateinischen oder mittelhochdeutschen Kleide.

In den ältesten deutschen Sprichwörtern gehören unter anderen: „Nicht alle Vögel sind Falken“; „Wer zwei Hasen zugleich jagt, fängt keinen.“ - "Hər oxuyan Molla Pənah olmaz”, "Bir əldə iki qarpız tutmaq olmaz" oder "iki qoçun başı bir qazanda qaynamaz".

$\mathrm{Zu}$ den Kennzeichen des Sprichwortes gehört seine seit altersfeststehende und von einer Generation auf die andere Form, die selbst für den Zeitgeist verbindlich ist. Hat ein Spruch hier und da einen doppelten Wortlaut, so handelt es sich um ganz unwesentliche, oft landschaftlich bedingte Abweichungen formeller ist.

Das oberste Stilgesetz des Sprichwortes ist die Kürze. Sehr häufig sind die Vierwort- Sprüche, wie z. B. ,jedem Tierchen sein Pläsierchen“ oder „At minənindir, q1lınc bağlayanın“, „İt hürər, karvan keçər“" u.s.w. Dreiwortsprüche sind im Aserbaidschanischen häufiger als im Deutschen „Getroffene Hunde bellen“ oder „Qoyunu qurda tapşırıb“, „Dəvəsi ölmüş ərəbəm“", „Ayıdan xarrat olmaz.“"

Beliebt ist ferner die Verbildlichung eines Gedankens durch Einführung eines Hauses oder Waldtieres. Am häufigsten treten Katze und Hund, sowie Wolf und Bär auf. Von den Haustieren spielen außerdem das Pferd, der Esel, das Schaf im aserbaidschanischen Sprichwort eine größere Rolle als im westeuropäischen.

Eine Sonderart bilden die aserbaidschanischen Sprichwörter, die Frage und Antwort zugleich enthalten und so einen dramatischen Charakter gewinnen: „İtə dedilər: -- Gündə neçə dəfə döyülürsən?” Dedi:"-Mərdimazara rast gəlməknəndir!“

Dəvəyə dedilər: ,--Boynun əyridir!“Dedi: ,-- Haram düzdür ki, boynum da düz ola?!“

Bei der Gegenüberstellung der Sprichwörter der beiden Völker taucht unter manchen anderen Fragen nach Charakter, Mäßigung, Temperament, Witz des einen oder anderen Volkes auch die Frage auf: Welche Menschen und welche menschlichen Eigenschaften sind es, die der Volkswitz und die Volkskritik am schärfsten angreifen? Die spitzen Pfeile des Sprichwortes, mag es, deutsch oder aserbaidschanisch sein, richten sich auf die gleichen Ziele: sie treffen die Faulen, die Dummen, die geizigen reichen, die herrischen Unterdrücken, die Ausbeuter, die Volksdummer, die betrügerische Händler.

\section{LITERATUR}

1. Barsuk R.Y. Some problems of foreign language teaching in Azerbaijani schools.

2. E. A. Ivanikova. The basis of the primitive phraseological units.

3. A. Huseynzade. Proverbs, Baku, 1949, 1956, 1981

4. Azerbaijan Philology Issues, 3rd edition, Baku, 1991

5. Azad Nabiyev, Proverbs and sayings, Baku, Chirag, 2009, I book. 